\title{
MONOMIALE DARSTELLUNG ENDLICHER GRUPPEN
}

\author{
BERTRAM HUPPERT
}

Eine wichtige Aufgabe der Darstellungstheorie ist die Aufstellung eines vollsčändigen Systems von inäquivalenten irreduziblen Darstellungen einer endlichen Gruppe (\$. Obwohl man Verfahren kennt, die dies theoretisch leisten (etwa die Reduktion der regulären Darstellung), ist die praktische Durchführung nur in wenigen Fällen möglich. Die Aufgabe vereinfacht sich jedoch wesentlich, wenn sich alle irreduziblen Darstellungen von $\mathbb{B}$ auf monomiale Gestalt transformieren lassen; denn alle monomialen Darstellungen einer endlichen Gruppe hann man nach einem sehr einfachen Verfahren gewinnen (siehe [4], S. 140). Liegt dieser Fall vor, so nennen wir $\$ 3$ im Anschluss an Itô [2] eine $M$-Gruppe. Wichtige Klassen von endlichen Gruppen sind $M$-Gruppen. Die erste Untersuchung dieser Art stammt wohl von Blichfeldt [3], der zeigte, dass jede Gruppe von Primzahlpotenzordnung eine $M$-Gruppe ist. Ein sehr allgemeines Kriterium ist das folgeride von Zassenhaus [7] herrührende: $\mathbb{B}$ is t eine $M$-Gruppe, wenn $\mathbb{G}$ einen abelschen Normalteiler $\Re$ besitzt, dessen Faktorgruppe $\mathscr{G} / \mathcal{Q}$ primzahlstufige Hauptreihen hat. Itô [2] gab kürzlich ein anderes, in der Zassenhausschen Aussage nicht enthaltenes Kriterium an; er zeigte, dass jede aúíüsbare Gruppe mit lauter abelschen Sylowgruppen eine $M$-Gruppe ist. Wir beweisen hier cinen Satz, der die Ergebnisse von Zassenhaus und Itô umfasst.

Satz. Die endliche Gruppe $\&$ besitze einen auflösbaren Normalteiler $?$, welcher lauter abelsche Sylowgruppen habe. $B / \Re$ sei eine Gruppe mit primzahlstufigen Hauptreihen. ${ }^{1)}$ Dann ist (3) eine $M$-Gruppe.

Beweis. Da sich die Voraussetzung auf jede Untergruppe von (S) überträgt, genügt der Nachweis eines abelschen Normalteilers von $\$$, der nicht im Zentrum $3(\$ 3)$ von $\$$ s liegt.

$\Re$ habe die Stuie $k$, d.h. es sei $\left.\Re^{(k-1)} \neq \mathfrak{E},\right\urcorner^{(k)}=\mathfrak{r}$. Nach Hall [1] oder Taunt [5] besitzt 9 einen eirdeutig bestimmten maximalen abelschen Normalteiler $\mathfrak{B}$, der sich darstellen lässt in der Gestalt

$$
\mathfrak{N}=3(\mathfrak{Y}) \times 3\left(\mathfrak{Y}^{\prime}\right) \times \ldots \times 3\left(\mathfrak{Y}^{(k-1)}\right) .
$$

$\$$ ist charalkteristische Untergruppe von $\%$, also Normalteiler von $\mathcal{G}$. Indem wir, falls notwendig, $\Re$ curch $\Re(\$(\$)$ ersetzen, können wir annehmen, dass $3(\$)$ in $?$ enthalien ist. Dann liegt $\mathfrak{Z}(\mathbb{B})$ in $\mathfrak{Z}(\mathfrak{Y})$, also auch in $\mathfrak{B}$. Ist $\mathfrak{B}$ von $\mathfrak{Z}(\mathbb{B})$ verschieden, so sind wir ferig. Aus $\mathfrak{Z}=3(\mathfrak{B})$ folgt $\mathfrak{B}=3(\mathfrak{Y})$ und $\mathfrak{l}^{\prime}=(\mathfrak{F}$ wegen

Received July 13, 1953.

1) Eine Gruppe mit prinzahlstufigen Hauptreihen wollen wir nach Zappa [6] als ülerauföshar bezeichnen. 
der Gestalt von $\mathfrak{N}$. Somit fallen $\mathfrak{B}, \mathfrak{\imath}$ und $\mathfrak{Z}(\mathfrak{B})$ zusammen. Da $\mathbb{\$} / \mathfrak{\Re}$ überauflösbar ist, existiert ein Normalteiler $\mathfrak{M}$ von $\mathbb{S}$ derart, dass $\mathfrak{M} / \mathfrak{N}$ Primzahlordnung hat. $\mathfrak{M}$ ist als zyklische Erweiterung des Zentrums abelsch und liegt gewiss nicht in $3(\mathbf{( S )})$. Damit ist der gewünschte Normalteiler in allen Fällen nachgewiesen.

Es sei noch vermerkt, dass jede endliche Gruppe genau einen minimalen Normalteiler mit überaufösbarer Faktorgruppe besitzt. Dies ergibt sich aus dem Folgenden:

Sind $\mathbb{S} / \mathfrak{M}$ und $\mathbb{B} / \mathfrak{N}$ überaufösbar, so auch $\mathbb{S} / \mathfrak{M} \cap \mathfrak{N}$.

Beweis. Ohne Beschränkung der Allgemeinheit sei $\mathfrak{M} \cap \mathfrak{N}=\mathfrak{F}$. Wir legen eine Hauptreihe durch $\mathbb{S} / \mathfrak{M}$ und $\mathfrak{M} \times \mathfrak{R} / \mathfrak{M}$ und betrachten deren $z$ wischen $\mathfrak{M} \times \mathfrak{R}$ und $\Re$ liegende Gruppen:

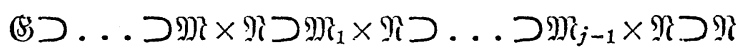

Setzen wir $\mathfrak{M}_{0}=\mathfrak{M}$ und $\mathfrak{M}_{j}=\mathfrak{E}$, so ist die Ordnung von $\mathfrak{M}_{i} / \mathfrak{M}_{i+1}$ eine Primzah für $i=0, \ldots, j . \mathfrak{M}_{i} \times \mathfrak{i}$ und $\mathfrak{M}$ sind Normalteiler von $\mathfrak{G}$, also auch $\mathfrak{M}_{i} \times \mathfrak{M} \frown \mathfrak{M}$ $=\mathfrak{M}_{i}$. Eine primzahlstufige Hauptreihe von $\mathfrak{B} / \mathfrak{M}$ lässt sich nun durch die $\mathfrak{M}_{i}$ zu einer primzahlstufigen Hauptreihe von $\$$ ergänzen.

Durch wiederholte Bildung des minimalen Normalteilers mit überaufösbarer Faktorgruppe kann man nun eine “absteigende überauflösbare Reihe" bilden. Die entsprechende aufsteigende Reihe existiert jedoch im allgemeinen nicht; denn die Vereinigung von zwei überauflösbaren Normalteilern einer Gruppe (\$) ist nicht notwendig überauflösbar. Dies zeigt das folgende Beispiel:

Wir erweitern eine elementar abelsche Gruppe $\mathfrak{P}=\left\{P_{1}, P_{2}\right\}$ der Ordnung 25 mit einer Quaternionengruppe, welche von den Elementen $A$ und $B$ erzeugt werde. $A$ und $B$ mögen in $\mathfrak{P}$ die folgenden Automorphismen bewirken:

$$
A P_{1} A^{-1}=P_{1}^{2}, A P_{2} A^{-1}=P_{2}^{3}, B P_{1} B^{-1}=P_{2}, B P_{2} B^{-1}=P_{1}^{-1} \text {. }
$$

Wie man leicht sieht, sind $\mathfrak{R}=\mathfrak{P} \cdot\{A\}$ und $\mathfrak{M}=\mathfrak{P} \cdot\{B\}$ überauflösbare Normalteiler der entstehenden Gruppe. Aber die Gruppe $\mathfrak{P}\{A, B\}$ ist nicht überauflösbar, da $\mathfrak{P}$ ein minimaler Normalteiler ist.

\section{LITERATUR}

[1] Hall, P. The construction of soluble groups, Journ. für reine u. angew. Math. 182, 206214, (1940).

[2] Itô, N. Note on A-groups, Nagoya Math. Journ. 4, 79-81, (1952).

[3] Miller-Blichfeldt-Dickson. Finite groups.

[4] Speiser, A. Theorie der Gruppen von endlicher Ordnung, 3. Aufl. 1937.

[5] Taunt, D. R. On A-groups, Proc. Cambridge philos. Soc. 45, 24-42. (1949).

[6] Zappa, G. Sui gruppi supersolubili, Rend. Sem. Mat. Univ. Roma (4) 2, 323-330, (1937).

[7] Zassenhaus, H. Über endliche Fastkörper, Abh. math. Seminar Hansischen Univ. 11, 187-220, (1936).

Mathematisches Institut

d?r Universität Tübingen 